\title{
Multi-infeed HVDC/AC power system modeling and analysis with dynamic phasor application
}

\author{
Haojun Zhu, Zexiang Cai, Haoming Liu and Yixin Ni, Senior Member, IEEE
}

\begin{abstract}
In this paper, dynamic phasor method has been applied to HVDC transmission system and FACTS device modeling. A hybrid-model simulation algorithm is suggested for transient stability analysis with HVDC transmission system and FACTS devices in dynamic phasor models and other ac system elements in electromechanical models. Computer test results show clearly that the proposed hybrid-model simulation algorithm can be applied for transient stability analysis effectively and accurately. A multi-infeed HVDC/AC (MIDC/AC) system is studied using the suggested hybrid-model transient stability program. The dynamic behaviors of MIDC/AC system under different circumstances are discussed. The characteristics of HVDC system recovery and recovery strategy for MIDC system are considered in the stability analysis. Furthermore, the impacts of advanced FACTS controllers such as STATCOM on system dynamic are studied for enhance system operation security.
\end{abstract}

Index Terms-- multi-infeed, HVDC transmission, dynamic phasor, transient stability, hybrid simulation

\section{INTRODUCTION}

$\mathrm{T}_{\mathrm{s}}^{\mathrm{h}}$ he overall dynamic behavior of large scale interconnected system is of great concern especially under large disturbances. Among them, the multi-infeed HVDC/AC (MIDC/AC) system is most complicated due to complex ACDC and DC-DC interactions. A lot of research efforts have been devoted to MIDC/AC system modeling, analysis, controlling so as to enhance bulk system stability.

There are basically two types of HVDC transmission system simulation programs. One is electromagnetic transient program (EMTP), which is time tedious in simulation with extremely small step-length and not suitable for transient stability study; the other is transient stability program with HVDC converter expressed in quasi-steady-state (QSS) model,

This work was jointly supported by the National Natural Science Foundation of China (50337010) and the Research Grant Council of Hong Kong SAR Government (CityU 1/00C).

Haojun Zhu is with the College of Electrical Engineering, South China University of Technology, Guangzhou, 510640, China (e-mail: zhuhaojun@vip.sina.com).

Zexiang Cai is with the College of Electrical Engineering, South China University of Technology, Guangzhou, 510640, China (e-mail: epzxcai@scut.edu.cn).

Yixin Ni is with the University of Hong Kong, Hong Kong, China (e-mail: yxni@eee.hku.hk). which is not suitable to consider complex converter faults or asymmetric ac-network faults close to dc converters. In recent years, a new and promising method --- dynamic phasor method [1] has been developed to study the effects of power electronic devices (PEDs), such as FACTS controllers and HVDC transmission systems. By truncating unimportant higher order components and keeping only those significant components, the dynamic phasor model is capable of retaining dominant dynamic features of PEDs. This approach has been successfully applied in modeling of certain FACTS devices, such as TCSC[2], UPFC[3] and ac machines[4].

In this paper, dynamic phasor method has been applied to HVDC transmission system and FACTS device such as STATCOM modeling. A hybrid-model simulation algorithm is suggested for transient stability analysis with HVDC transmission system and FACTS devices in dynamic phasor models and other ac system elements in electromechanical models. Computer test results show clearly that the suggested HVDC transmission and FACTS devices dynamic phasor models can catch up dynamic characteristics of HVDC transmission and FACTS devices very well as compared with their EMT models. And the transient stability simulation results of a two-area DC/AC interconnected power system show that using the suggested interface method can be integrated into conventional transient stability program effectively and accurately.

A multi-infeed HVDC/AC (MIDC/AC) system is studied using the suggested hybrid-model transient stability program. The dynamic behaviors of MIDC/AC system under different circumstances are discussed. The characteristics of HVDC system recovery and recovery strategy for MIDC system are considered in the stability analysis. Furthermore, the impacts of advanced FACTS controllers such as STATCOM on system dynamic are studied for enhance system operation security.

\section{OUTLINES OF THE DYNAMIC PHASOR MODEL}

\section{A. Dynamic Phasor Model of HVDC System}

An HVDC transmission power system is composed of rectifier, inverter, and dc transmission line. Fig. 1. is a schematic diagram of a single-pole HVDC system. 


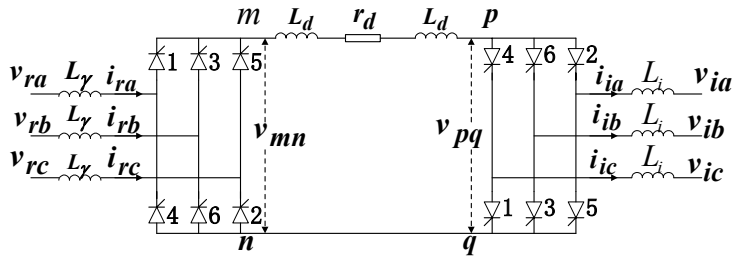

Fig. 1. Schematic diagram of HVDC

Using switching functions represent the three conditions for each valve in HVDC converters (turning on, turning off and commutation), the time-domain model of rectifier of HVDC system can be described as:

$$
\begin{gathered}
v_{m n}=v_{d r}=\left(v_{r a} S_{r v 1}+v_{r b} S_{r v 3}+v_{r c} S_{r v 5}\right) \\
\quad-\left(v_{r a} S_{r v 4}+v_{r b} S_{r v 6}+v_{r c} S_{r v 2}\right) \\
i_{r a}=i_{1}-i_{4}=i_{d} S_{r i 1}-i_{d} S_{r i 4} \\
i_{r b}=i_{3}-i_{6}=i_{d} S_{r i 3}-i_{d} S_{r i 6} \\
i_{r c}=i_{5}-i_{2}=i_{d} S_{r i 5}-i_{d} S_{r i 2} \\
\left(2 L_{d}\right) \frac{d i_{d}}{d t}+r_{d} i_{d}=v_{d r}-v_{d i}
\end{gathered}
$$

$V_{d r}$ is the dc voltage of the rectifier terminal; $i_{r a}, i_{r b}, i_{r c}$ are the ac currents of the rectifier terminal; $S_{r v 1}, S_{r v 2}, \ldots, S_{r v 6}$ are switching functions corresponding to the voltages of the rectifier valves $V_{1} \sim V_{6}$, and $S_{r i 1}, S_{r i 2}, \ldots, S_{r i 6}$ are switching functions corresponding to the currents of the rectifier valves $V_{1} \sim V_{6}$. The switching function of valve $V_{1}$ is shown as example in (2) and (3), the other switch functions are lagged by $3 / \pi$ each.

$$
\begin{aligned}
& S_{r v 1}= \begin{cases}\frac{1}{2} & {[\alpha+2 k \pi, \alpha+\gamma+2 k \pi]} \\
1 & {\left[\alpha+\gamma+2 k \pi, \alpha+\frac{2 \pi}{3}+2 k \pi\right]} \\
\frac{1}{2} & {\left[\alpha+\frac{2 \pi}{3}+2 k \pi, \alpha+\gamma+\frac{2 \pi}{3}+2 k \pi\right]} \\
0 & {\left[\alpha+\gamma+\frac{2 \pi}{3}+2 k \pi, \alpha+2(k+1) \pi\right]}\end{cases} \\
& S_{r i 1}= \begin{cases}\frac{\theta-\alpha}{\gamma} & {[\alpha+2 k \pi, \alpha+\gamma+2 k \pi]} \\
1 & {\left[\alpha+\gamma+2 k \pi, \alpha+\frac{2 \pi}{3}+2 k \pi\right]} \\
1-\frac{\theta-\alpha-2 \pi / 3}{\gamma} & {\left[\alpha+\frac{2 \pi}{3}+2 k \pi, \alpha+\gamma+\frac{2 \pi}{3}+2 k \pi\right]} \\
0 & {\left[\alpha+\gamma+\frac{2 \pi}{3}+2 k \pi, \alpha+2(k+1) \pi\right]}\end{cases}
\end{aligned}
$$

where $\gamma$ is the commutation angle, and $\alpha$ is the lagging firing-angle. When the Valve is commutating, we assume commutation current is changing with slopes of $1 / \gamma$ and $-1 / \gamma$, and $S_{r v 1}=0.5$ for commutation voltage[5].

Then we further assume that its ac-side variables can be described accurately enough by their fundamental frequency components $(k=1)$; and its dc-side variables can be described accurately enough by the dc component. Hence for the switching functions both $\mathrm{dc}$ and fundamental frequency components should be retained. For simplicity supposing ac system is under three phases balanced condition, hence dynamic phasor of $S_{r v 1}$ and $S_{r i 1}$ can be used to express the HVDC rectifier model, and the inverter can be handled in the same way:

$$
\begin{array}{rlrl}
I_{r a 1}^{r} & =2 I_{d 0} S_{r i 1,1}^{r} & I_{i a 1}^{r}=2 I_{d 0} S_{i i 1,1}^{r} \\
I_{r a 1}^{i}=2 I_{d 0} S_{r i 1,1}^{i} & I_{i a 1}^{i}=2 I_{d 0} S_{i i 1,1}^{i} \\
V_{d r 0}=12 \operatorname{Re}\left[V_{r a 1} S_{r v 1,1}^{*}\right] & V_{d i 0}=-12 \operatorname{Re}\left[V_{i a 1} S_{i v 1,1}^{*}\right]
\end{array}
$$

$\frac{d I_{d 0}}{d t}=\frac{1}{2 L_{d}}\left[V_{d r 0}-V_{d i 0}-r_{d} I_{d 0}\right]$

Where dynamic phasors are be separated into the real and imaginary parts (the superscripts $r$ and i represent real part and imaginary part; the subscripts $r$ and $i$ represent rectifier and inverter). Dynamic phasors for switching functions $S_{r v 1}$ and $S_{r i 1}$ can be obtained through Fourier series formula.

\section{B. Dynamic phasor Model of STATCOM}

A STATCOM(see Fig.2) consists of a solid-state voltage source converters (VSC) and a DC link capacitor. VSC realizes constant voltage control of $V_{S}$ and $V_{d} ; m_{E}$ and $\delta_{E}$ are modulation ratio of PWM control and firing angle of VSC. And the basic relations of them are :

$$
\left\{\begin{array}{l}
V_{E}=m_{E} V_{d} \\
\theta_{E}=\theta_{S}-\delta_{E}
\end{array}\right.
$$
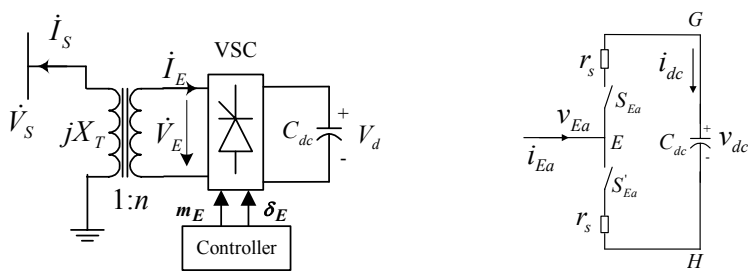

Fig. 2. Diagram of STATCOM Fig. 3. Equivalent circuit of STATCOM

Taking phase a as the reference phase, Fig. 3 shows the equivalent circuit of phase a with its valve switching simulated by ideal switch-state functions $S_{E a}$ and $S_{E a}^{\prime}$ $\left(S_{E a}+S_{E a}^{\prime}=1\right)$. It is clear that $v_{E a}$ and $i_{E a}$ have the relation (' $n$ ' is the ac system neutral point)

$$
\begin{aligned}
v_{E a} & =v_{E a H}+v_{H n} \\
& =\left[\left(i_{E a} r_{s}+v_{d c}\right) \cdot S_{E a}+\left(i_{E a} r_{s}\right) \cdot S_{E a}^{\prime}\right]+v_{H n}
\end{aligned}
$$

For a balanced ac system, it is easy to derive

$$
v_{H n}=-\frac{1}{3} v_{d c} \sum_{j=a, b, c} S_{E j}
$$

Substituting (9) into (8), we have

$$
v_{E a}=i_{E a} r_{s}+v_{d c} \cdot S_{E a}-\frac{1}{3} v_{d c} \sum_{j=a, b, c} S_{E j}
$$

The dc capacitor dynamics can be described as

$$
C_{d c} \frac{d v_{d c}}{d t}=i_{d c}=i_{E d c}-i_{B d c}=\sum_{j=a, b, c}\left(i_{E j} S_{E j}-i_{B j} S_{B j}\right)
$$

The switch-state functions $S_{E a}, S_{E a}^{\prime}$ are determined by the PWM control, we can only filter out the fundamental wave component and dc component of the switching functions $S_{E a}$ [6], which can be expressed as 


$$
d_{E j}=\frac{m_{E}}{2} \cos \left(\omega t-\delta_{E}-\sigma_{j}\right)+\frac{1}{2}
$$

where, $j=a, b, c$, and $\sigma_{a}=0, \sigma_{b}=\frac{2}{3} \pi, \sigma_{c}=\frac{4}{3} \pi$.

Keeping fundamental frequency components $(k=1)$ and dc component $(k=0)$ in (8) and (9), and separating real and imaginary parts, we obtain the dynamic phasor model of STATCOM:

$$
\left\{\begin{array}{c}
\left\langle V_{E a}\right\rangle_{1}^{r}=\left\langle I_{E a}\right\rangle_{1}^{r} \cdot r_{s}+\left\langle V_{d c}\right\rangle_{0} \cdot \frac{m_{E}}{4} \cos \theta_{E} \\
\left\langle V_{E a}\right\rangle_{1}^{i}=\left\langle I_{E a}\right\rangle_{1}^{i} \cdot r_{s}+\left\langle V_{d c}\right\rangle_{0} \cdot \frac{m_{E}}{4} \sin \theta_{E} \\
\frac{d\left\langle V_{d c}\right\rangle_{0}}{d t}=\frac{m_{E}}{C_{d c}} \frac{3}{2}\left[\left\langle I_{E a}\right\rangle_{1}^{r} \cdot \cos \theta_{E}+\left\langle I_{E a}\right\rangle_{1}^{i} \cdot \sin \theta_{E}\right]
\end{array}\right.
$$

\section{HYBRID-MODEL SIMULATION ALGORITHM}

\section{A. General Interface Algorithm}

A general hybrid-model interface algorithm for HVDC system and FACTS devices is proposed. The system is divided into two parts in time simulation. One is HVDC and FACTS subsystem, which is modeled in dynamic phasor; the other is the remaining part including generators, loads and ac network, which is described in conventional phasor models, as shown in Fig. 4.

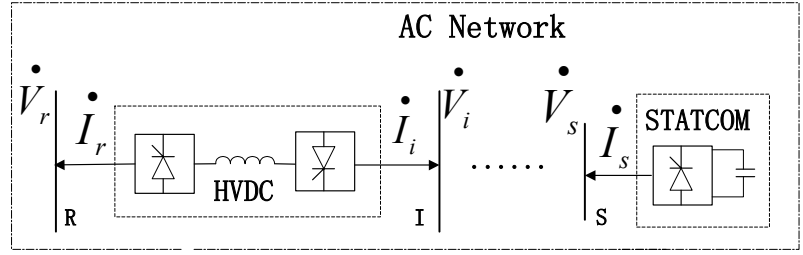

Fig. 4. Interface of HVDC and FACTS with network

For the HVDC and FACTS subsystem, ac system is equivalent to voltage sources at the interface buses, while for the ac network subsystem, HVDC and FACTS system is equivalent to injection current sources. At each time step, the two subsystems are solved through iteration based on Newton Raphson method explained below for better convergence.

Supposing the bus admittance matrix of ac/dc system are reduced to generator internal buses and interface buses remained, and the corresponding reduced bus admittance matrix equation takes the form:

$$
\left[\begin{array}{ll}
Y_{11} & Y_{12} \\
Y_{21} & Y_{22}
\end{array}\right]\left[\begin{array}{l}
\dot{V}_{G} \\
\dot{V}_{C}
\end{array}\right]=\left[\begin{array}{l}
\dot{I}_{G} \\
\dot{I}_{C}
\end{array}\right]
$$

Here, $\dot{V}_{G}, \dot{I}_{G}$ are generator internal bus voltage and injection current. And $\dot{V}_{C}=\left[\begin{array}{lll}\dot{V}_{r} & \dot{V}_{i} & \dot{V}_{s}\end{array}\right]^{T}, \dot{I}_{C}=\left[\begin{array}{lll}\dot{I}_{r} & \dot{I}_{i} & \dot{I}_{s}\end{array}\right]^{T}$ are interface ac bus voltage and injection current respectively. Then:

$$
\dot{I}_{C}=Y_{21} \dot{V}_{G}+Y_{22} \dot{V}_{C}
$$

Deduced from dynamic phasor model (4) and (11), we can obtain $\dot{I}_{D P}$, which is the equivalent injection current from HVDC and FACTS system to ac system. And it should be equal to $\dot{I}_{C}$ in (13):

$$
\Delta \dot{I}_{C}=\dot{I}_{C}-\dot{I}_{D P}=0
$$

The mismatch equation (14) is functions of interface bus voltage $\dot{V}_{C}$. Newton-Raphson algorithm is applied to solve (14) till convergence. The detailed iteration steps are as follow assuming $\dot{V}_{G}$ is known from integration of generator state variables:

1) Initialize the voltages of converter ac buses $V_{C}^{(0)}, \theta_{C}^{(0)}$ (set iteration number $l=0)$;

2) Calculate $\dot{I}_{C}$ according to (13) and $\dot{I}_{D P}$ according to (4) and (11);

3) If $\Delta \dot{I}_{C}$ is less than the given tolerance, then $V_{C}^{(l)}$ and $\theta_{C}^{(l)}$ are considered as the final solution for the time step, otherwise go to step 4;

4) Calculate Jacobian matrix $J_{a c}$ of (14), and calculate $\Delta \dot{V}_{c}$ through $\Delta \dot{V}_{C}=-J_{a c}^{-1} * \dot{\Delta}_{C}$;

5) Update $\dot{V}_{C}^{(l+1)}=\dot{V}_{C}^{(l)}+\Delta \dot{V}_{C}, l=l+1$, and go to step 2 .

\section{B. Computer Results}

A test dynamic phasor model time-domain simulation, using MATLAB/SIMULINK toolbox, are compared with the corresponding EMT model. Here, Fig. 5(a) shows DC current of EMT HVDC model; Fig. 5(b) shows DC current of dynamic phasor HVDC model; Fig. 5(c) shows AC current of rectifier; Fig. 5(d) is DC voltage of capacitor in STATCOM. And dot line is result of dynamic phasor model and solid line is result of EMT model.

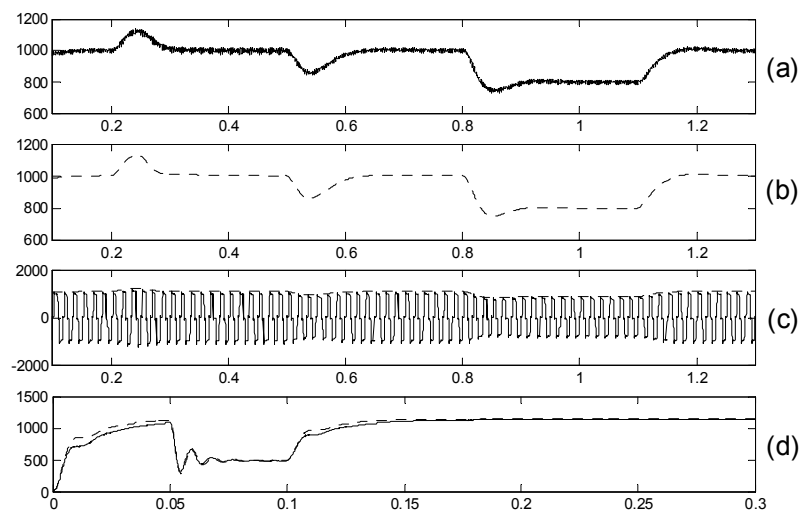

Fig. 5. Comparison of dynamic phasor and EMT model

Simulation results under disturbance demonstrate obviously that dynamic phasor models have very good accuracy as compared with corresponding EMT models. And they are far more efficient than EMT ones. For example, dynamic phasor HVDC model simulation costs $2.2 \mathrm{~s}$, which is much less than $15.4 \mathrm{~s}$ that EMT HVDC model simulation costs.

Furthermore, a 4-generator 2-area interconnected power system (see Fig. 6) is used for the computer test, which is implemented by Power System Toolbox in MATLAB.

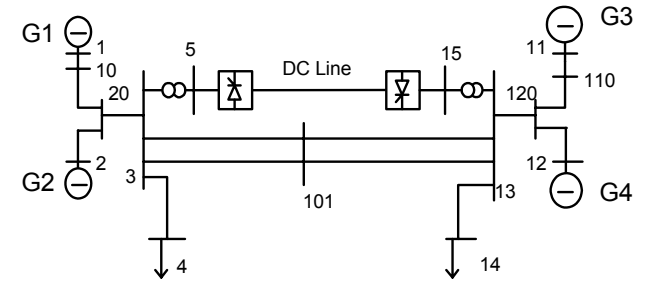


Fig. 6. 4 generators 2 areas test system

Two cases are studied: (i) QSS model is used for HVDC system(solid line); (ii) dynamic phasor model is used for HVDC system(dot line). Fig. 7 (a) and (b) shows voltage magnitude of bus 5; Fig. 7(c) and 7(d) shows generator angle of $\mathrm{G} 4$ relative to $\mathrm{G} 1$. All results demonstrate good consistency of these two models, and simulation time of these two model are almost equal when both are at the same simulation time step, which proves that the suggested hybrid-model simulation algorithm works accurately and effectively in transient stability analysis. The unique advantage of proposed model lies in that the behaviors of converter bridges can be well described by switch functions, which makes the model have the capability to consider HVDC system impacts on overall system transient stability in better precision relative to QSS model.

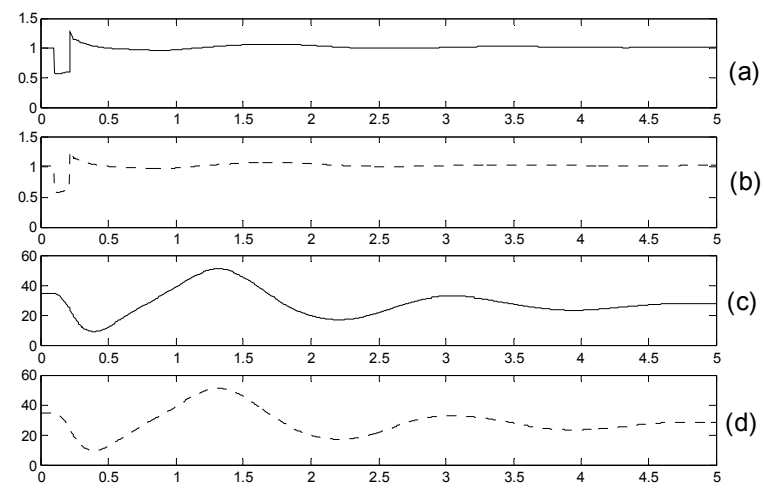

Fig. 7. Results comparison of two models

\section{MIDC/AC SYSTEM DYNAMIC CHARACTERISTICS}

Among MIDC systems, security issues arise most easily in system where ac system is relatively weak due to large amount of feeding power delivered by HVDC systems. In this paper, dynamic characteristics of this category of MIDC system are discussed only.

The study case is based on a simplified diagram of South China Grid, which is one of the most complex MIDC/AC systems. As is shown in Fig. 8, three HVDC systems and three ac transmission corridors delivery large amounts power from area A1, A2, A3 to a load centralized area A4. The suggested hybrid-model simulation is applied for analysis.

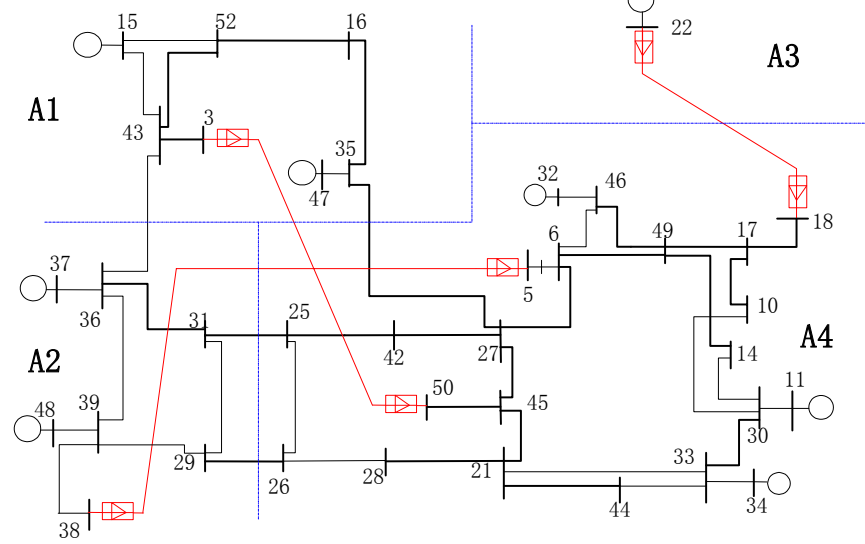

Fig. 8. Simplified diagram of South China Grid

\section{A. Effective Short Circuit Ratio (ESCR)}

The concept of effective short circuit ratio has been used to calculate the ESCR for two base cases. For each base case, ESCR for each inverter bus were calculated. The fault current $\mathrm{I}_{\mathrm{F}}$ was measured as the initial fault current after applying a bus fault. The simulations results are summarized in Table I:

Table I ESCR (SB=100MVA)

\begin{tabular}{|c|c|c|c|c|c|}
\hline Case & \multirow{2}{*}{$\begin{array}{c}\text { Pdc } \\
(\mathrm{MW})\end{array}$} & $\begin{array}{c}\text { Qdc } \\
(\mathrm{MVAr})\end{array}$ & Bus & $\mathrm{I}_{\mathrm{F}}(\mathrm{pu})$ & ESCR \\
\hline \multirow{2}{*}{2004} & \multirow{3}{*}{4893} & \multirow{2}{*}{1727} & 5 & 104.43 & 1.71 \\
\cline { 4 - 6 } & & & 50 & 158.53 & 2.81 \\
\hline \multirow{2}{*}{2005} & \multirow{2}{*}{7174} & \multirow{2}{*}{2696} & 50 & 176.95 & 3.20 \\
\cline { 4 - 6 } & & & 18 & 128.42 & 1.41 \\
\cline { 4 - 6 } & & & & &
\end{tabular}

Case 2004 and 2005 ESCR are calculated by ( $\left.\mathrm{I}_{\mathrm{F}^{-}} \mathrm{Qdc}\right) / \mathrm{Pdc}$. In both case ESCR of bus 5 are smallest, which means that bus 5 is the weakest point. In Case 2005, total dc power is Pdc $=71.74 \mathrm{MW}$ (78 pu) with the summation of capacity in inverters bus is Qdc $=26.96 \mathrm{MVAr}$, and all ESCR are below 2, which means this multi-infeed HVDC/AC transmission system is very weak at each inverter side in case 2005. Further research should be made to improve system stability, especially voltage stability and reactive power support.

\section{B. Transient Stability Analysis}

Because three inverter buses are electrical in close proximity in this system, disturbance at any inverter bus will dynamically interact other inverter buses. For example, when line 6--27 occurs three phases grounding fault, simulations results show that voltage at bus 5 drops to 0 pu while voltage of bus 50 and 18 also drop to $0.4 \mathrm{pu}$ and $0.6 \mathrm{pu}$, and that may lead to inverters occur commutation failure. The most severe situation is that all three inverter are bypassing during fault period. To study transient stability under this situation, three phases grounding fault at each buses of area A4 are simulated, and fault is clear after $0.1 \mathrm{~s}$ by tripping corresponding line. Simulation results of fault at bus 6 are shown in Fig. 9. Fig. 9(a) shows de current of bus 5 , which is bypass in $0.1 \mathrm{~s}-0.2 \mathrm{~s}$ and recovers quickly in 50ms after fault clearing. Fig. 9(b) is rotor angle of Gen 48 with respect to Gen 34, indicating system remain stable. But if any of three HVDC systems is unable to recover normally, the system will be unstable without generators tripping, as rotor angle of Gen 48 with respect to Gen 34 shown in Fig 9(c).

Here are some conclusions from simulations results. 1) three phase grounding fault at most of buses in receiving end system will lead to low voltage two or three inverters during fault occurring. 2) if fault is clear in $0.1 \mathrm{~s}$ and all HVDC systems restart quickly(in $50 \mathrm{~ms}$ ), the system remain stable. 3) If any HVDC system fails to recover, system will be unstable without generator tripping. 4) Fast recovery of HVDC system is essential to overall system stability. 

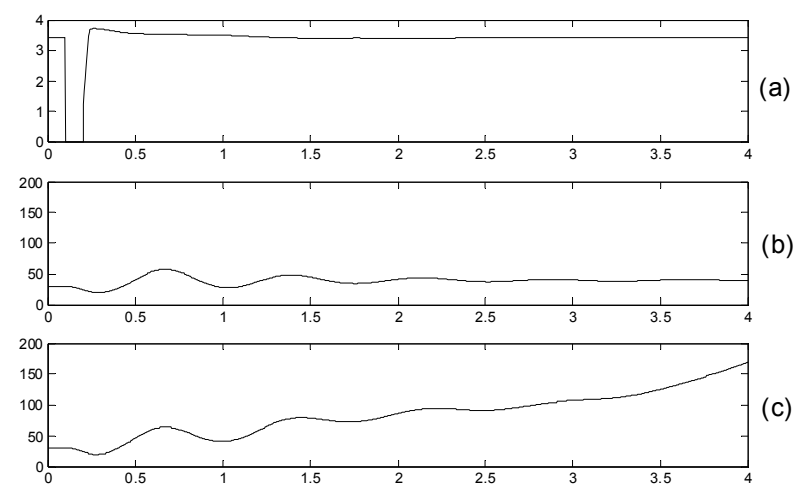

Fig. 9. Result of fault at line 6--27

\section{HVDC System Recovery Study}

HVDC system recovery has great influence on dynamic performance of connected ac system, while HVDC system recovery speed also depends on strength of ac system. In case 2005, a three-phase fault occurs near inverter bus is used to study the dynamic performance with different recover times (very fast: $50 \mathrm{~ms}$, fast : $300 \mathrm{~ms}$ and slow : $600 \mathrm{~ms}$ ). The results are shown in Fig. 10. De current of HVDC system 40-12 is shown in Fig. 10(a); Fig. 10(b) demonstrates voltage of inverter bus 12; Fig. 10(c) shows rotor angle of Gen 48 with respect to Gen 34 (solid line: very fast recovery; dash line: fast recovery; dot line: slow recovery).
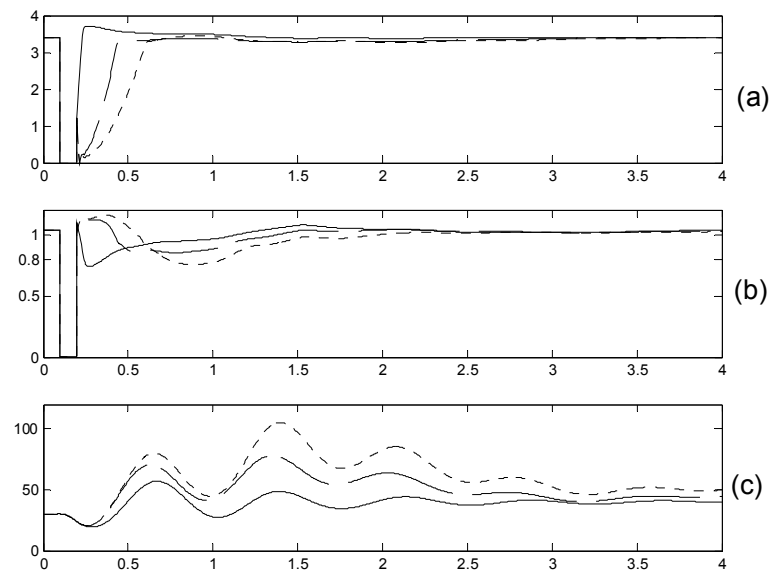

Fig. 10. Dynamic response of different recover times

Generally speaking, the faster of the HVDC recover rate, the faster the system can return to stable. From the result, we can see that, if all the three HVDC is recovering too fast (in $50 \mathrm{~ms}$ ), the ac voltage will drop significantly during recovering period because it required large amounts of reactive power. If the HVDC systems are recovering too slow (in $600 \mathrm{~ms}$ ), the impact to ac system is greater. To avoid further commutation failure, medium recovery speed (in $300 \mathrm{~ms}$ ) is more appropriate for smooth voltage and less impact.

In multi-infeed HVDC system, a staggered recovery strategy [7] can be applied to achieve optimal overall performance. For example, one of HVDC system (38-5) is recovering fast to reduce the impact, and the other two HVDC systems (3-50 and 22-18) are in slower recovery speed to ease the demand of reactive power. Fig. 11 shows improved performance of the staggered recovery strategy (dot line) comparing with routine recovery (solid line), where Fig. 11(a) is voltage at bus 12 and Fig. 11(b) is rotor angle of Gen 48 with respect to Gen 34 .
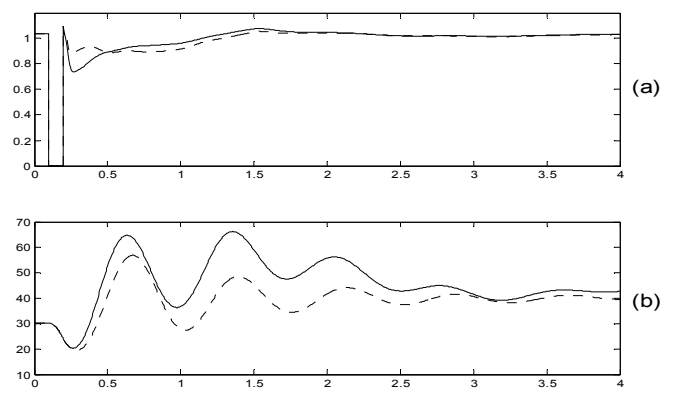

Fig. 11. Dynamic performance of staggered recovery strategy

\section{Dynamic Reactive Power Support}

Fast recovery of HVDC system is important to system security situation, but it is limited by reactive power demand. One of the compensation means -- fixed capacitor banks are usually employed, but they reduce ac system strength and their performance in fast recovery is unsatisfied. A dynamic reactive power support means - STATCOM is introduced for fast recovery of multi-infeed HVDC system. Here STATCOM is set at inverter bus 5, and its control diagram is shown in Fig. 12 , in which constant ac terminal bus voltage control is achieved by controlling $m_{E}$ of the PWM controller, and the constant dc link capacitor voltage control realized by controlling the firing angle $\delta_{E}$. Fast recovery of HVDC system with STATCOM (dot line) and with capacitor compensation (solid line) are compared in Fig. 13. STATCOM is obviously superior to capacitor compensation in recovery performance because of its dynamic reactive power compensation characteristics.

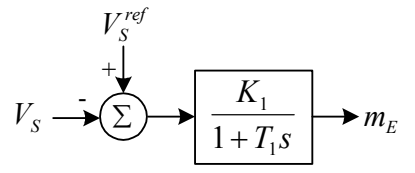

(a)

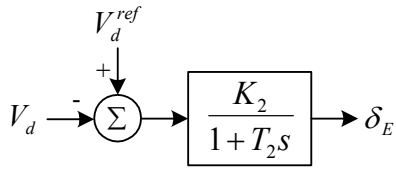

(b)
Fig. 12. The control system of STATCOM

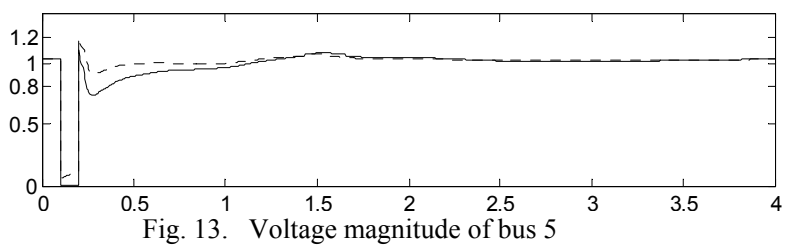

\section{CONCLUSIONS}

1) Dynamic phasor method has been applied to HVDC transmission system and STATCOM modeling.

2) A general hybrid-model transient stability simulation algorithm for DC/AC system with FACTS devices is suggested, which is proved to 
be effective and accurate compared with conventional transient stability program.

3) Fast recovery of HVDC system is essential to security situation of MIDC system.

4) Staggered recovery strategy and dynamic reactive power support by STATCOM are capable to improve dynamic performance of overall system.

\section{REFERENCES}

[1] S. R. Sanders, J. M. Noworolski, X. Z. Liu, et al. "Generalized averaging method for power conversion circuits," IEEE Trans. on Power Electronics, vol. 6, pp. 251-259, Apr. 1991.

[2] A. M. Stankovic, P. Mattavelli, V. Caliskan, et al. "Modeling and analysis of FACTS devices with dynamic phasors," in Proc. 2000 IEEE Power Engineering Society Winter Meeting, pp 1440-1446

[3] P.C. Stefannov, A. M. Stankovic, "Modeling of UPFC operation under unbalanced conditions with dynamic phasors, "IEEE Trans on Power Systems, vol. 17, pp. 395-403, May. 2002

[4] A. M. Stankovic, S. R. Sanders, "Dynamic phasors in modeling and analysis of unbalanced polyphase ac machines, "IEEE Trans on Energy Conversion, vol. 17, pp. 107-113, Mar. 2002

[5] A. R. Wood, J. Arrillaga, "HVDC convertor waveform distortion: a frequency-domain analysis, "IEE Proc. Gener. Transm. Distrib., vol. 142, pp. 88-96,1995

[6] N. Nabavi and M. E. Iravani, "Steady-state and dynamic models of unified power flow controllers (UPFC) for power system studies," IEEE Trans. Power Systems, vol. 11, pp. 1937-1943, Nov. 1996.

[7] J. Reeve, S. P. lane-smith, "Multi-infeed HVDC transient response and recovery strategies," IEEE Trans. on Power Delivery, vol. 8, pp. 19952001, Oct. 1993.

\section{BIOGRAPHIES}

Haojun Zhu was born in Guangdong, China, in Feb. 1978. He received his B.S. degree, M.S. degree from South China University of Technology (SCUT), China in 2001 and 2003 respectively. He is currently a graduate student for Ph.D. degree in the College of Electrical Engineering of SCUT with research interests in power system modeling, stability and control and HVDC transmission.

Zexiang Cai was born in Nanjing, China, in July 1960. He received his B.S. degree from Huainan Mineral Institute in 1982, M.S. degree from Northeast China Institute of Electrical Power Engineering in 1985, Ph.D. degree from Tsinghua University in 1991. He has been a professor of the College of Electrical Engineering of the South China University of Technology since 1998. His research interests are power system protection relay, power system stability and control.

Haoming Liu was born in Jiangsu, China, in Feb, 1977. He received his B. Eng., M. Eng., and Dr. Eng. from Nanjing University of Science and Technology, China in 1998, 2001 and 2003 respectively. His research interests are power system stability and control and electricity markets. Currently, he is a post-doctor at the Department of Electrical Engineering, Southeast University, China.

Yixin Ni (SM'94) received her B. Eng., M. Eng., and Dr. Eng. All from the Department of Electrical Engineering, Tsinghua University, China in 1968, 1981 and 1983 respectively. Her research interests are power system stability and control, HVDC transmission, FACTS, and electricity markets. She was a professor of Tsinghua University and is now with the University of Hong Kong. She is a recipient of several nation-wide awards. 